TRANSACTIONS OF THE

AMERICAN MATHEMATICAL SOCIETY

Volume 362, Number 3, March 2010, Pages 1301-1324

S 0002-9947(09)04960-5

Article electronically published on October 20, 2009

\title{
ASYMPTOTIC CURVATURE DECAY AND REMOVAL OF SINGULARITIES OF BACH-FLAT METRICS
}

\author{
JEFFREY STREETS
}

\begin{abstract}
We prove a removal of singularities result for Bach-flat metrics in dimension 4 under the assumption of bounded $L^{2}$-norm of curvature, bounded Sobolev constant and a volume growth bound. This result extends the removal of singularities result for special classes of Bach-flat metrics obtained by Tian and Viaclovsby. For the proof we emulate Cheeger and Tian and analyze the decay rates of solutions to the Bach-flat equation linearized around a flat metric. This classification is used to prove that Bach-flat cones are in fact ALE of order 2. This result is then used to prove the removal of singularities theorem.
\end{abstract}

\section{INTRODUCTION}

In dimension 4, the Euler-Lagrange equations for the functional

$$
\mathcal{W}(g):=\int_{M}\left|\mathrm{~W}_{g}\right|^{2} d V_{g},
$$

where $\mathrm{W}_{g}$ is the Weyl curvature, are given by

$$
B_{i j}=\nabla^{k} \nabla^{l} W_{i k j l}+\frac{1}{2} R^{k l} W_{i k j l}=0,
$$

where $W_{i j k l}$ and $R^{k l}$ correspond to the components of the Weyl and Ricci tensors, respectively [4] 6]. $B_{i j}$ are the components of the Bach tensor, and a metric which is critical for $\mathcal{W}$ is called Bach-flat.

A smooth Riemannian manifold $\left(X^{4}, g\right)$ is called an asymptotically locally Euclidean end of order $\tau$ if there exists a finite subgroup $\Gamma \subset S O(4)$ which acts freely on $\mathbb{R}^{4} \backslash B(0, R)$ and a $C^{\infty}$ diffeomorphism $\phi: X \rightarrow\left(\mathbb{R}^{4} \backslash B(0, R)\right) / \Gamma$ such that, using this identification,

$$
\begin{aligned}
g_{i j} & =\delta_{i j}+O\left(r^{-\tau}\right), \\
\partial^{|k|} g_{i j} & =O\left(r^{-\tau-k}\right),
\end{aligned}
$$

for any partial derivative of order $k$ as $r \rightarrow \infty$. We say an end is ALE of order 0 if there exist coordinates as above so that

$$
\begin{aligned}
g_{i j} & =\delta_{i j}+o(1), \\
\partial^{|k|} g_{i j} & =o\left(r^{-k}\right),
\end{aligned}
$$

as $r \rightarrow \infty$.

Received by the editors September 11, 2007.

2000 Mathematics Subject Classification. Primary 53C25.

(C)2009 American Mathematical Society Reverts to public domain 28 years from publication 
Theorem 1.1. Let $\left(X^{4}, g\right)$ be a complete, noncompact four-dimensional Bach-flat space with zero scalar curvature which is ALE of order 0. Assume further that

$$
\int_{X}\left|\operatorname{Rm}_{g}\right|^{2} d V_{g}<\Lambda<\infty, \quad C_{S}<K<\infty .
$$

Then $(X, g)$ is ALE of order 2 .

This theorem is an extension of Theorem 1.1 of [9], which gives the ALE order 0 structure. In particular, given a noncompact Bach-flat space with zero scalar curvature, under the same hypotheses on curvature and Sobolev constant and also assuming $b_{1}(X)<\infty$, where $b_{1}(X)$ is the first Betti number, it is proved that there are finitely many ends of order zero.

Theorem 1.2. Let $(X, d, x)$ be a complete locally compact length space with base point $x$. Let $B(x, 1) \backslash\{x\}$ be a connected $C^{\infty}$ Bach-flat constant scalar curvature four-manifold satisfying

$$
\begin{aligned}
& \int_{B(x, 1)}\left|\operatorname{Rm}_{g}\right|^{2} d V_{g}<\infty, \\
& \|u\|_{L^{4}(B(x, 1) \backslash\{x\})} \leq C_{s}|| \nabla u \|_{L^{2}(B(x, 1) \backslash\{x\})}, \quad u \in C^{0,1}(B(x, 1) \backslash\{x\}), \\
& b_{1}(X)<\infty,
\end{aligned}
$$

where $C_{s}$ is a positive constant. Then the metric $g$ extends to $B(x, 1)$ as a smooth orbifold metric.

This is an extension of Theorem 6.4 of [8], and indeed this result was suggested in the work of Gang Tian and Jeff Viaclovsky [8, 9]. There the $C^{0}$ orbifold structure of such singularities is proved in general and smoothness of the orbifold singularities is proved for certain subclasses of Bach-flat manifolds, specifically half-conformally flat metrics, metrics with harmonic curvature, and constant scalar curvature Kähler metrics. The proof uses certain Kato inequalities which are satisfied in these special cases to improve the decay of the Ricci tensor, which in turn can be used to get improved decay of the full curvature tensor. The techniques used herein also suffice to give this result assuming that the metric has harmonic curvature, that is,

$$
\delta \mathrm{Rm}=-\nabla^{i} R_{i j k l}=0 .
$$

Finally we point out that the assumption on $b_{1}(X)$ is required to get an upper bound on the volume growth of the manifold. This is quite subtle and is spelled out in the work of [9]. The theorem holds if one instead assumes such an upper bound directly.

We now give an outline of the rest of the paper. In section 2 we decompose the action of the bi-Laplacian acting on symmetric two-tensors according to the radial separation of variables on a cone. In section 3 we compute the linearization of the Bach-flat condition around a flat metric. We observe that after applying a change of diffeomorphism gauge and possibly a conformal change this equation is equivalent to the equation $\Delta^{2} h=0$, where $h$ is the perturbation of the metric. Using the separation of variables we further simplify this equation and classify the solutions in terms of their decay or growth rates at infinity. Section 4 gives the proof of Theorem 1.1, which consists of considering the Bach-flat equation at infinity in the cone as a perturbation of a flat metric, and using our analysis of the linearized equation. Section 5 uses Theorem 1.1 to prove Theorem 1.2 . 


\section{DeCOMposition OF THE Bi-LAPLACIAN}

In this section we will decompose the action of the bi-Laplacian on symmetric two-tensors on $\mathbb{R}^{4}$ according to the cone structure. These tensors naturally decompose according to radial and tangential directions. We reintroduce the notation of [1] and recall the decomposition of the Laplacian proved there. These lemmas are then used in a straightforward way to decompose the action of the bi-Laplacian. The calculation is greatly simplified by restricting to $\mathbb{R}^{4}$, where we completely understand the curvature tensor, and how it decomposes according to the cone structure.

Write $\left(\mathbb{R}^{4}, g_{\text {flat }}\right)$ as $C\left(S^{3}, g_{\text {can }}\right)$, the cone over the three-sphere with its canonical metric. Let $P: T\left(1, S^{3}\right) \rightarrow T\left(r, S^{3}\right)$ denote the identification of tangent bundles induced by parallel transport along radial geodesics, and let $\widetilde{\nabla}^{r}$ denote the Riemannian connection with respect to the induced metric on $\left(r, S^{3}\right)$. Define the connection

$$
\underline{\widetilde{\nabla}}=P \widetilde{\nabla}^{1} P^{-1} .
$$

A basic formula is then

$$
\widetilde{\nabla}^{r}=r^{-1} \underline{\tilde{\nabla}} .
$$

Also let $\widetilde{d^{r}}, \widetilde{\delta}^{r}, \widetilde{\operatorname{tr}}^{r}$ be defined with respect to the induced metric on $\left(r, S^{3}\right)$ and let $\widetilde{d^{r}}$ denote exterior differentiation on $\left(r, S^{3}\right)$. Then define

$$
\begin{aligned}
\underline{\widetilde{\delta}} & =P \widetilde{\delta}^{1} P^{-1}, \\
\underline{\tilde{d}} & =P \widetilde{d}^{1} P^{-1}, \\
\underline{\tilde{\mathrm{tr}}} & =P \widetilde{\operatorname{tr}}^{1} P^{-1} .
\end{aligned}
$$

The following formulae are immediate:

$$
\begin{aligned}
\widetilde{\Delta}^{r} & =r^{-2} \widetilde{\underline{\Delta}}, \\
\widetilde{\delta}^{r} & =r^{-1} \widetilde{\widetilde{\delta}}, \\
\widetilde{d}^{r} & =r^{-1} \widetilde{\underline{d}}, \\
\widetilde{\operatorname{tr}}^{r} & =\underline{\widetilde{\operatorname{tr}}} .
\end{aligned}
$$

Now say $e$ is a vector tangent to $\left(r, S^{3}\right)$ and $e^{*}$ is its dual 1-form. Let $\eta$ be a 1 -form such that

$$
\begin{gathered}
\eta\left(\frac{\partial}{\partial r}\right)=0 \\
\nabla_{\frac{\partial}{\partial r}} \eta \equiv 0 .
\end{gathered}
$$

Some simple formulas are then:

$$
\begin{aligned}
\nabla_{e} \eta & =r^{-1}\left(\underline{\widetilde{\nabla}}_{e} \eta-\eta(e) d r\right), \\
\nabla_{e} d r & =r^{-1} e^{*}, \\
\nabla_{\frac{\partial}{\partial r}} \frac{\partial}{\partial r} & =\nabla_{\frac{\partial}{\partial r}} d r=0 .
\end{aligned}
$$


Now fix $\frac{\partial}{\partial r}, e_{1}, e_{2}, e_{3}$ a local orthonormal basis satisfying

$$
\widetilde{\nabla}_{e_{i}}^{r} e_{j}=0
$$

at a fixed point $(r, x)$ and also

$$
\nabla_{\frac{\partial}{\partial r}} e \equiv 0
$$

Finally, given $\omega_{1}, \omega_{2} 1$-forms let

$$
\omega_{1} \otimes \omega_{2}=\omega_{1} \otimes \omega_{2}+\omega_{2} \otimes \omega_{1} .
$$

Using this frame, the symmetric two-tensors naturally decompose into three distinct types. In the next four lemmas we record the action of divergence and the Laplacian on these tensors. We will need these preliminary calculations to calculate the action of the bi-Laplacian on each of these types of tensors. The proofs of these lemmas can all be found in 1 .

Lemma 2.1. Consider $f(r) B$, where $B=\sum \eta_{i} \otimes \eta_{j}$ and $\eta_{i}$ satisfies (2.5) and (2.6). Then

$$
\begin{aligned}
\nabla^{*} \nabla(f B)= & \left(-f^{\prime \prime}-3 r^{-1} f^{\prime}+r^{-2} f\left(\widetilde{\nabla}^{*} \widetilde{\widetilde{\nabla}}+2\right)\right) B \\
& +2 r^{-2} f \underline{\widetilde{\delta}}(B) \otimes d r-2 r^{-2} f \underline{\mathrm{tr}}(B) d r \otimes d r .
\end{aligned}
$$

Lemma 2.2. Consider $k(r) \tau \otimes d r$, where $\tau$ satisfies (2.5) and (2.6). Then

$$
\begin{aligned}
\nabla^{*} \nabla(k \tau \otimes d r)= & -2 r^{-2} k \underline{\widetilde{\nabla}}^{\text {sym }} \tau \\
& +\left\{\left[-k^{\prime \prime}-3 r^{-1} k^{\prime}+r^{-2} k\left(\widetilde{\widetilde{\nabla}}^{*} \underline{\nabla}+6\right)\right] \tau\right\} \otimes d r \\
& +4 r^{-2} k \underline{\tilde{\delta}} \tau d r \otimes d r,
\end{aligned}
$$

where

$$
\underline{\widetilde{\nabla}}^{\mathrm{sym}} \tau=\sum_{i} \widetilde{\nabla}_{e_{i}} \tau \otimes e_{i}^{*} .
$$

Lemma 2.3. Consider $l(r) \phi(x) d r \otimes d r$. Then

$$
\begin{aligned}
\nabla^{*} \nabla(l \phi d r \otimes d r)= & -2 r^{-2} l \phi \underline{\widetilde{g}}-2 r^{-2} l \underline{\tilde{d}} \phi \otimes d r \\
& +\left(-l^{\prime \prime}-3 r^{-1} l^{\prime}+r^{-2} l\left(\underline{\widetilde{\nabla}}^{*} \underline{\widetilde{\nabla}}+6\right)\right) \phi d r \otimes d r .
\end{aligned}
$$

Lemma 2.4. For tensors of the three types considered above, we have the following formulas:

$$
\begin{aligned}
\delta(f B) & =r^{-1} f \underline{\widetilde{\delta}}(B)-r^{-1} f \underline{\underline{\operatorname{tr}}}(B) d r, \\
\delta(k \tau \otimes d r) & =k^{\prime} \tau+4 r^{-1} k \tau+r^{-1} k(\widetilde{\widetilde{\delta}} \tau) d r, \\
\delta(l \phi d r \otimes d r) & =\left(l^{\prime}+3 r^{-1} l\right) \phi d r .
\end{aligned}
$$


Proposition 2.5. Consider $f(r) B$, where $B=\sum \eta_{i} \otimes \eta_{j}$ and $\eta_{i}$ satisfies (2.5) and (2.6). Then

$$
\begin{aligned}
\left(\nabla^{*} \nabla\right)^{2}(f B)= & \left(f^{(4)}+6 r^{-1} f^{(3)}-r^{-2} f^{\prime \prime}\left(1+2 \underline{\widetilde{\nabla}}^{*} \underline{\widetilde{\nabla}}\right)\right) B \\
& -\left(r^{-3} f^{\prime}\left(2 \widetilde{\widetilde{\nabla}}^{*} \widetilde{\nabla}+7\right)\right) B+r^{-4} f\left(\left(\underline{\widetilde{\nabla}}^{*} \widetilde{\widetilde{\nabla}}+2\right)\right)^{2} B \\
& -4 r^{-4} f \widetilde{\nabla}^{\mathrm{sym}} \widetilde{\delta}(B)+4 r^{-4} f \underline{\widetilde{\mathrm{tr}}}(B) \underline{\underline{g}} \\
& +4\left\{\left[-r^{-2} f^{\prime \prime}-r^{-3} f^{\prime}+r^{-4} f\left(\widetilde{\widetilde{\nabla}}^{*} \underline{\widetilde{\nabla}}+2\right)\right] \widetilde{\delta}(B)\right\} \otimes d r \\
& +8 r^{-4} f \underline{\widetilde{d \operatorname{tr}}}(B) \otimes d r \\
& +4\left(r^{-2} f^{\prime \prime}+r^{-3} f^{\prime}-r^{-4} f\left(\widetilde{\nabla}^{*} \underline{\nabla}+4\right)\right) \underline{\operatorname{tr}}(B) d r \otimes d r \\
& +8 r^{-4} f \underline{\widetilde{\delta}}(B) d r \otimes d r .
\end{aligned}
$$

Proof. This will be a straightforward calculation using the three lemmas above. First of all using (2.13) we see that

$$
\begin{aligned}
-\nabla^{*} \nabla\left(f^{\prime \prime} B\right)= & \left(f^{(4)}+3 r^{-1} f^{(3)}-r^{-2} f^{\prime \prime}\left(\underline{\widetilde{\nabla}}^{*} \underline{\widetilde{\nabla}}+2\right)\right) B \\
& -2 r^{-2} f^{\prime \prime} \underline{\widetilde{\delta}}(B) \otimes d r+2 r^{-2} f^{\prime \prime} \underline{\widetilde{\mathrm{tr}}}(B) d r \otimes d r .
\end{aligned}
$$

Next

$$
\begin{aligned}
\nabla^{*} \nabla\left(-3 r^{-1} f^{\prime} B\right)= & 3\left(r^{-1} f^{(3)}+r^{-2} f^{\prime \prime}-r^{-3} f^{\prime}\left(\widetilde{\widetilde{\nabla}}^{*} \underline{\widetilde{\nabla}}+3\right)\right) B \\
& -6 r^{-3} f^{\prime} \underline{\widetilde{\delta}}(B) \otimes d r+6 r^{-3} f^{\prime} \underline{\widetilde{\mathrm{t}}}(B) d r \otimes d r .
\end{aligned}
$$

Now it is clear that $\widetilde{\nabla}^{*} \widetilde{\nabla} B$ satisfies (2.5) and (2.6). Thus apply (2.13) again to compute

$$
\begin{aligned}
\nabla^{*} \nabla & \left(r^{-2} f\left(\underline{\widetilde{\nabla}}^{*} \underline{\vec{\nabla}}+2\right) B\right) \\
= & \left(-r^{-2} f^{\prime \prime}+r^{-3} f^{\prime}+r^{-4} f\left(\widetilde{\widetilde{\nabla}}^{*} \widetilde{\nabla}+2\right)\right)\left(\widetilde{\widetilde{\nabla}}^{*} \underline{\nabla}+2\right)(B) \\
& +2 r^{-4} f\left(\underline{\widetilde{\nabla}}^{*} \widetilde{\nabla}-2\right)(\underline{\widetilde{\delta}} B) \otimes d r+4 r^{-4} f \underline{\widetilde{d} \widetilde{\mathrm{tr}}} B \otimes d r \\
& -2 r^{-4} f\left(\widetilde{\widetilde{\nabla}}^{*} \widetilde{\nabla}+2\right) \underline{\operatorname{tr}}(B) d r \otimes d r .
\end{aligned}
$$

Note that we have applied Lemma 6.2 to commute $\underline{\widetilde{\delta}}$ past $\widetilde{\nabla}^{*} \underline{\widetilde{\nabla}}$. Now, since $\eta$ satisfies (2.5) and (2.6), so does $\underline{\tilde{\delta}} B$; thus we may apply (2.14) to conclude

$$
\begin{aligned}
\nabla^{*} \nabla & \left(2 r^{-2} f \underline{\widetilde{\delta}}(B) \otimes d r\right) \\
= & -4 r^{-4} f \underline{\widetilde{\nabla}}^{\mathrm{sym}} \underline{\widetilde{\delta}}(B) \\
& +\left\{\left[-2 r^{-2} f^{\prime \prime}+2 r^{-3} f^{\prime}+2 r^{-4} f\left(\underline{\widetilde{\nabla}}^{*} \underline{\widetilde{\nabla}}+6\right)\right] \underline{\widetilde{\delta}}(B)\right\} \otimes d r \\
& +8 r^{-4} f \underline{\delta \widetilde{\delta}}(B) d r \otimes d r .
\end{aligned}
$$


Similarly we apply (2.16) to conclude

$$
\begin{aligned}
\nabla^{*} \nabla & \left(-2 r^{-2} f \underline{\widetilde{\operatorname{tr}}}(B) d r \otimes d r\right) \\
= & 4 r^{-4} f \underline{\widetilde{\operatorname{tr}}}(B) \underline{\widetilde{g}}+4 r^{-4} f \underline{\widetilde{d \operatorname{tr}}}(B) \otimes d r \\
& +\left(2 r^{-2} f^{\prime \prime}-2 r^{-3} f^{\prime}-2 r^{-4} f\left(\widetilde{\nabla}^{*} \widetilde{\nabla}+6\right)\right) \underline{\widetilde{\operatorname{tr}}}(B) d r \otimes d r .
\end{aligned}
$$

Summing together (2.21)-(2.25) gives the result.

Proposition 2.6. Consider $k(r) \tau \otimes d r$, where $\tau$ satisfies (2.5) and (2.6). Then

$$
\begin{aligned}
& \left(\nabla^{*} \nabla\right)^{2}(k \tau \otimes d r) \\
& =4\left(r^{-2} k^{\prime \prime}+r^{-3} k^{\prime}-r^{-4} k\left(\underline{\widetilde{\nabla}}^{*} \underline{\widetilde{\nabla}}+5\right)\right)\left(\underline{\widetilde{\nabla}}^{\mathrm{sym}} \tau\right) \\
& -8 r^{-4} k \underline{\widetilde{\delta}} \tau \underline{\widetilde{g}} \\
& +\left(k^{(4)}+6 r^{-1} k^{(3)}-r^{-2} k^{\prime \prime}\left(2 \underline{\widetilde{\nabla}}^{*} \underline{\widetilde{\nabla}}+9\right)-r^{-3} k^{\prime}\left(2 \underline{\widetilde{\nabla}}^{*} \underline{\widetilde{\nabla}}+15\right)\right. \\
& \left.+r^{-4} k\left(\left(\underline{\widetilde{\nabla}}^{*} \underline{\nabla}\right)^{2}+16 \underline{\widetilde{\nabla}^{*}} \underline{\underline{\nabla}}+28\right)\right) \tau \otimes d r \\
& -12 r^{-4} k \underline{\widetilde{d \delta}} \tau \otimes d r \\
& +8\left(-r^{-2} k^{\prime \prime}-r^{-3} k^{\prime}+r^{-4} k\left(\underline{\widetilde{\nabla}}^{*} \underline{\widetilde{\nabla}}+6\right)\right) \underline{\tilde{\delta}} \tau d r \otimes d r .
\end{aligned}
$$

Proof. We must start from the expression in (2.14) and apply (2.13), (2.14), and (2.16) to the individual terms. First of all it is clear that if $\tau$ satisfies (2.5) and (2.6), then so does $\underline{\nabla}^{\text {sym }} \tau$. Thus we may apply (2.13) to conclude

$$
\begin{aligned}
\left(\nabla^{*} \nabla\right) & \left(-2 r^{-2} k \underline{\nabla}^{\text {sym }} \tau\right) \\
= & \left(2 r^{-2} k^{\prime \prime}-2 r^{-3} k^{\prime}-2 r^{-4} k\left(\widetilde{\nabla}^{*} \underline{\widetilde{\nabla}}+2\right)\right)\left(\underline{\widetilde{\nabla}}^{\text {sym }} \tau\right) \\
& +4 r^{-4} k\left(\underline{\widetilde{\nabla}}^{*} \underline{\widetilde{\nabla}}-\underline{\widetilde{d} \tilde{\delta}}-2\right) \tau \otimes d r+8 r^{-4} k \underline{\widetilde{\delta}} \tau d r \otimes d r,
\end{aligned}
$$

where we have used the equations

$$
\underline{\widetilde{\delta}}\left(\underline{\widetilde{\nabla}}^{\mathrm{sym}} \tau\right)=-\underline{\widetilde{\nabla}}^{*} \underline{\widetilde{\nabla}} \tau+\underline{\widetilde{d} \widetilde{\delta}} \tau+2 \tau, \quad \underline{\widetilde{\operatorname{tr}}}\left(\underline{\widetilde{\nabla}}^{\text {sym }} \tau\right)=2 \underline{\widetilde{\delta}} \tau
$$

from Lemma 6.2. Now we apply (2.14) to conclude

$$
\begin{aligned}
\left(\nabla^{*} \nabla\right) & \left(-k^{\prime \prime} \tau \otimes d r\right) \\
= & 2 r^{-2} k^{\prime \prime} \underline{\nabla}^{\mathrm{sym}} \tau \\
& +\left\{\left[k^{(4)}+3 r^{-1} k^{(3)}-r^{-2} k^{\prime \prime}\left(\widetilde{\nabla}^{*} \underline{\nabla}+6\right)\right] \tau\right\} \otimes d r \\
& -4 r^{-2} k^{\prime \prime} \underline{\tilde{\delta}} \tau d r \otimes d r .
\end{aligned}
$$

Similarly,

$$
\begin{aligned}
\left(\nabla^{*} \nabla\right) & \left(-3 r^{-1} k^{\prime} \tau \otimes d r\right) \\
= & 6 r^{-3} k^{\prime} \underline{\nabla}^{\mathrm{sym}} \tau \\
& +3\left\{\left[r^{-1} k^{(3)}+r^{-2} k^{\prime \prime}-r^{-3} k^{\prime}\left(\widetilde{\nabla}^{*} \underline{\widetilde{\nabla}}+7\right)\right] \tau\right\} \otimes d r \\
& -12 r^{-3} k^{\prime} \underline{\tilde{\delta}} \tau d r \otimes d r .
\end{aligned}
$$


Again,

$$
\begin{aligned}
\left(\nabla^{*} \nabla\right) & \left(r^{-2} k\left(\underline{\widetilde{\nabla}}^{*} \underline{\nabla}+6\right) \tau\right) \otimes d r \\
= & -2 r^{-4} k\left(\widetilde{\widetilde{\nabla}}^{*} \underline{\nabla}+8\right) \underline{\widetilde{\nabla}}^{\mathrm{sym}} \tau \\
& +\left\{\left[-r^{-2} k^{\prime \prime}+r^{-3} k^{\prime}+r^{-4} k\left(\underline{\widetilde{\nabla}}^{*} \underline{\widetilde{\nabla}}+6\right)\right]\left(\underline{\widetilde{\nabla}}^{*} \underline{\widetilde{\nabla}}+6\right) \tau\right\} \otimes d r \\
& +4 r^{-4} k\left(\widetilde{\widetilde{\nabla}}^{*} \underline{\nabla}+4\right) \underline{\widetilde{\delta}} \tau d r \otimes d r,
\end{aligned}
$$

where we commuted derivatives using Lemma 6.2 Finally using (2.16) with $l=$ $r^{-2} k$ and $\phi=\underline{\tilde{\delta}} \tau$ we compute

$$
\begin{aligned}
\left(\nabla^{*} \nabla\right) & \left(4 r^{-2} k \underline{\widetilde{\delta}} \tau\right) d r \otimes d r \\
= & -8 r^{-4} k \underline{\widetilde{\delta}} \tau \underline{\widetilde{g}}-8 r^{-4} k \underline{\widetilde{d} \widetilde{\delta}} \tau \otimes d r \\
& +4\left[-r^{-2} k^{\prime \prime}+r^{-3} k^{\prime}+r^{-4} k\left(\widetilde{\nabla}^{*} \underline{\widetilde{\nabla}}+6\right)\right](\underline{\widetilde{\delta}} \tau) d r \otimes d r .
\end{aligned}
$$

Collecting together (2.27)-(2.31) gives the result.

Proposition 2.7. Consider $l(r) \phi(x) d r \otimes d r$. Then

$$
\begin{aligned}
\left(\nabla^{*} \nabla\right)^{2}( & l \phi d r \otimes d r) \\
= & 4\left(r^{-2} l^{\prime \prime}+r^{-3} l^{\prime}-r^{-4} l\left(\underline{\widetilde{\nabla}}^{*} \underline{\widetilde{\nabla}}+4\right)\right)(\phi \underline{\widetilde{g}}) \\
& +4 r^{-4} l \underline{\widetilde{\nabla}^{\mathrm{sym}} \tilde{\tilde{d}} \phi} \\
& +4\left(r^{-2} l^{\prime \prime}+r^{-3} l^{\prime}-r^{-4} l\left(\underline{\widetilde{\nabla}}^{*} \underline{\widetilde{\nabla}}+8\right)\right) \underline{\tilde{d}} \phi \otimes d r \\
& +\left(l^{(4)}+6 r^{-1} l^{(3)}-r^{-2} l^{\prime \prime}\left(2 \underline{\widetilde{\nabla}}^{*} \underline{\widetilde{\nabla}}+9\right)-r^{-3} l^{\prime}\left(2 \widetilde{\widetilde{\nabla}}^{*} \underline{\nabla}+15\right)\right. \\
& \left.\quad+r^{-4} l\left(\left(\underline{\widetilde{\nabla}}^{*} \underline{\nabla}\right)^{2}+20 \underline{\widetilde{\nabla}}^{*} \underline{\widetilde{\nabla}}+48\right)\right) \phi d r \otimes d r .
\end{aligned}
$$

Proof. We need to compute the action of the Laplacian of the right hand side of (2.16). First of all it is clear that $\phi \underline{g}$ satisfies (2.5) and (2.6). Thus we may apply (2.13) to conclude

$$
\begin{aligned}
\left(\nabla^{*} \nabla\right) & \left(-2 r^{-2} l \phi \underline{\widetilde{g}}\right) \\
= & \left(2 r^{-2} l^{\prime \prime}-2 r^{-3} l^{\prime}-2 r^{-4} l\left(\widetilde{\nabla}^{*} \underline{\widetilde{\nabla}}+2\right)\right)(\phi \underline{\widetilde{g}}) \\
& -4 r^{-4} l \underline{\widetilde{d}} \phi \otimes d r+12 r^{-4} l \phi d r \otimes d r,
\end{aligned}
$$

where we used that $\underline{\tilde{\delta}} \underline{\tilde{g}}=0$ and $\underline{\tilde{\mathrm{tr}}} \underline{\tilde{g}}=3$. Next we note that $\underline{\tilde{d}} \phi$ satisfies (2.5) and (2.6) so that we can apply (2.14) to conclude

$$
\begin{aligned}
\left(\nabla^{*} \nabla\right) & \left(-2 r^{-2} l\right) \underline{\tilde{d}} \phi \otimes d r \\
= & 4 r^{-4} l \underline{\widetilde{\nabla}}^{\mathrm{sym}} \underline{\underline{d}} \phi \\
& +\left\{\left[2 r^{-2} l^{\prime \prime}-2 r^{-3} l^{\prime}-2 r^{-4} l\left(\underline{\widetilde{\nabla}}^{*} \underline{\widetilde{\nabla}}+6\right)\right] \underline{\widetilde{d}} \phi\right\} \otimes d r \\
& +8 r^{-4} l \underline{\widetilde{\nabla}^{*}} \underline{\widetilde{\nabla}} \phi d r \otimes d r,
\end{aligned}
$$


where we have used the equation $\underline{\widetilde{\nabla}}^{*} \underline{\widetilde{\nabla}}=-\underline{\widetilde{\delta}}$. Next using (2.16) we compute

$$
\begin{aligned}
\left(\nabla^{*} \nabla\right) & \left(-l^{\prime \prime} \phi d r \otimes d r\right) \\
= & 2 r^{-2} l^{\prime \prime} \phi \underline{\widetilde{g}}+2 r^{-2} l^{\prime \prime} \underline{\widetilde{d}} \phi \otimes d r \\
& +\left(l^{(4)}+3 r^{-1} l^{(3)}-r^{-2} l^{\prime \prime}\left(\widetilde{\widetilde{\nabla}}^{*} \underline{\nabla}+6\right)\right) \phi d r \otimes d r,
\end{aligned}
$$

and similarly

$$
\begin{aligned}
\left(\nabla^{*} \nabla\right) & \left(-3 r^{-1} l^{\prime} \phi d r \otimes d r\right) \\
= & 6 r^{-3} l^{\prime} \phi \underline{\widetilde{g}}+6 r^{-3} l^{\prime} \underline{\widetilde{d}} \phi \otimes d r \\
& \left.+3\left(r^{-1} l^{(3)}+r^{-2} l^{\prime \prime}-r^{-3} l^{\prime}\left(\underline{\widetilde{\nabla}}^{*} \underline{\widetilde{\nabla}}+7\right)\right)\right) \phi d r \otimes d r .
\end{aligned}
$$

Finally we apply (2.16) to conclude

$$
\begin{aligned}
\left(\nabla^{*} \nabla\right) & \left(r^{-2} l\left(\underline{\widetilde{\nabla}}^{*} \underline{\widetilde{\nabla}}+6\right) \phi d r \otimes d r\right) \\
= & -2 r^{-4} l\left[\left(\widetilde{\widetilde{\nabla}}^{*} \underline{\nabla}+6\right) \phi\right] \underline{\tilde{g}}-2 r^{-4} l\left[\left(\underline{\widetilde{\nabla}}^{*} \widetilde{\widetilde{\nabla}}+8\right) \underline{\widetilde{d}} \phi\right] \otimes d r \\
& +\left(-r^{-2} l^{\prime \prime}+r^{-3} l^{\prime}+r^{-4} l\left(\underline{\widetilde{\nabla}}^{*} \underline{\widetilde{\nabla}}+6\right)\right)\left[\left(\widetilde{\widetilde{\nabla}}^{*} \underline{\widetilde{\nabla}}+6\right) \phi\right] d r \otimes d r .
\end{aligned}
$$

Collecting together (2.33)-(2.37) gives the result.

\section{The LinEARIZED EQUATION}

In this section we derive the equation for the linearization of the Bach-flat condition at a flat metric. The equation is of course not strictly elliptic due to the diffeomorphism invariance of the Bach-flat equation. After restricting to the case where the variation of the metric is trace-free and divergence-free, we are reduced to the biharmonic equation. We can then use the decomposition of the bi-Laplacian computed in the previous section to classify solutions to this equation and moreover compute the exact decay rates of solutions, which is the key ingredient of our removal of singularities result.

Proposition 3.1. Given g a flat metric, the linearization of the Bach-flat equation at $g$ is equivalent to the equation

$$
\begin{aligned}
0= & \Delta^{2} h-2 \Delta \delta^{*} \delta h \\
& +\frac{1}{3}\left(\nabla^{2} \Delta \operatorname{tr}_{g} h-\left(\Delta^{2} \operatorname{tr}_{g} h\right) g+\left(\Delta \delta^{2} h\right) g+2 \nabla^{2} \delta^{2} h\right) .
\end{aligned}
$$

Proof. Let $g(s)$ be a family of metrics such that $g(0)=g$ and $\frac{\partial}{\partial s} g(s)_{\mid s=0}=h$. First of all, since the metric is flat, it is clear that

$$
\left(\frac{1}{2} R^{k l} W_{i k j l}\right)^{\prime} \cdot h=0 .
$$

As a consequence of the Bianchi identity, in dimension 4 we have the equation

$$
\nabla^{k} \nabla^{l} W_{i k j l}=\Delta A_{i j}-\nabla^{k} \nabla_{i} A_{j k}
$$

where $A_{i j}$ are the components of the Weyl-Schouten tensor

$$
A=\frac{1}{n-2}\left(\mathrm{Rc}-\frac{1}{2(n-1)} R \cdot g\right) \text {. }
$$


A further application of the Bianchi identity then yields

$$
\nabla^{k} \nabla^{l} W_{i k j l}=\frac{1}{2}\left(\Delta \mathrm{Rc}_{i j}-\frac{1}{6} \Delta R g_{i j}-\frac{1}{3} \nabla_{i} \nabla_{j} R\right) .
$$

First we compute the linearization

$$
(\Delta \mathrm{Rc})^{\prime} \cdot h=-\frac{1}{2} \Delta\left(\Delta h+\nabla^{2} \operatorname{tr} h-2 \delta^{*} \delta h\right),
$$

and next

$$
\left.\left(-\frac{1}{6} \Delta R g\right)^{\prime} \cdot h=\frac{1}{6} \Delta(\Delta \operatorname{tr} h-\delta \delta h)\right) g
$$

Finally

$$
\left.\left(-\frac{1}{3} \nabla^{2} R\right)^{\prime} \cdot h=\frac{1}{3} \nabla^{2}(\Delta \operatorname{tr} h-\delta \delta h)\right)
$$

and the result follows.

Now, by a diffeomorphism gauge-fixing procedure, which we will use in the applications, we may assume that $h$ comes to us almost divergence-free. In fact we cannot prescribe that $h$ is exactly divergence-free. We will address this difficulty later in this section. Also, because of the conformal invariance of the Bach-flat equation, the trace of the right hand side of (3.1) vanishes. Thus there is no a priori separate equation that the trace of $h$ must satisfy. Using that $g$ has zero scalar curvature though yields that $h$ must satisfy

$$
\Delta \operatorname{tr} h=0 .
$$

As it turns out we can now remove the trace entirely by adding a term $L_{X} g$ for some vector field $X$. This corresponds to solving for a vector field $X$ satisfying

$$
\delta L_{X} g=0
$$

to preserve the divergence-free condition and

$$
\operatorname{tr}\left(L_{X} g\right)=2 \delta X=u,
$$

where $u$ is a prescribed harmonic function. It is shown in [1, lines 4.11-4.20] that on a Ricci-flat cone one can indeed solve for this $X$. Thus a divergence-free solution to (3.1) satisfying (3.2) can be written as $L_{X} g+h$, where

$$
\begin{aligned}
& 0=\Delta^{2} h, \\
& 0=\operatorname{tr}_{g} h, \\
& 0=\delta h .
\end{aligned}
$$

We now proceed to analyse solutions to (3.5). Let $h=f(r) B+k(r) \tau \otimes d r+$ $l(r) \phi(x) d r \otimes d r$. First of all the equation $\operatorname{tr} h=0$ reduces to the equation

$$
f \underline{\widetilde{\operatorname{tr}}}(B)+l \phi=0 \text {. }
$$

Next, using Lemma 2.4 and (3.6) the equation $\delta h=0$ reduces to the equations

$$
\begin{aligned}
r^{-1} f \underline{\widetilde{\delta}}(B)+\left(k^{\prime}+4 r^{-1} k\right) \tau & =0, \\
\left(l^{\prime}+4 r^{-1} l\right) \phi+r^{-1} k(\underline{\widetilde{\delta}} \tau) & =0 .
\end{aligned}
$$


Now we would like to write down and simplify the vertical component of equation (3.5). Using Propositions 2.20, 2.26 and 2.32 we see that this equation gives

$$
\begin{aligned}
0= & \left(l^{(4)}+6 r^{-1} l^{(3)}-r^{-2} l^{\prime \prime}\left(2 \underline{\widetilde{\nabla}}^{*} \widetilde{\widetilde{\nabla}}+9\right)-r^{-3} l^{\prime}\left(2 \underline{\widetilde{\nabla}}^{*} \underline{\widetilde{\nabla}}+15\right)\right. \\
& \left.+r^{-4} l\left(\left(\underline{\widetilde{\nabla}}^{*} \underline{\widetilde{\nabla}}\right)^{2}+20 \underline{\widetilde{\nabla}}^{*} \underline{\nabla}+48\right)\right) \phi \\
& +8\left(-r^{-2} k^{\prime \prime}-r^{-3} k^{\prime}+r^{-4} k\left(\underline{\widetilde{\nabla}}^{*} \underline{\widetilde{\nabla}}+6\right)\right) \underline{\widetilde{\delta}} \tau \\
& +4\left(r^{-2} f^{\prime \prime}+r^{-3} f^{\prime}-r^{-4} f\left(\underline{\widetilde{\nabla}}^{*} \underline{\nabla}+4\right)\right) \underline{\operatorname{tr}}(B) \\
& +8 r^{-4} f \underline{\widetilde{\delta}}(B) .
\end{aligned}
$$

Now we use equations (3.6), (3.7) and (3.8) to simplify this further. First of all, using (3.6), it is clear that

$$
\begin{aligned}
& 4\left(r^{-2} f^{\prime \prime}+r^{-3} f^{\prime}-r^{-4} f\left(\underline{\widetilde{\nabla}}^{*} \underline{\widetilde{\nabla}}+4\right)\right) \underline{\operatorname{tr}}(B) \\
& \quad=-4\left(r^{-2} l^{\prime \prime}+r^{-3} l^{\prime}-r^{-4} l\left(\underline{\widetilde{\nabla}}^{*} \widetilde{\nabla}+4\right)\right) \phi .
\end{aligned}
$$

Next, using (3.8), we compute

$$
\begin{aligned}
& 8\left(-r^{-2} k^{\prime \prime}-r^{-3} k^{\prime}+r^{-4} k\left(\underline{\widetilde{\nabla}}^{*} \underline{\widetilde{\nabla}}+6\right)\right) \underline{\tilde{\delta}} \tau \\
& \quad=8\left(r^{-1} l^{(3)}+7 r^{-2} l^{\prime \prime}-r^{-3} l^{\prime}\left(\underline{\widetilde{\nabla}}^{*} \underline{\widetilde{\nabla}}+1\right)-4 r^{-4} l\left(\underline{\widetilde{\nabla}}^{*} \underline{\widetilde{\nabla}}+6\right)\right) \phi .
\end{aligned}
$$

Finally using both (3.7) and (3.8) we compute

$$
\begin{aligned}
8 r^{-4} f \underline{\widetilde{\delta} \tilde{\delta}}(B) & =-8\left(r^{-3} k^{\prime}+4 r^{-4} k\right) \underline{\tilde{\delta}} \tau \\
& =8\left(r^{-2} l^{\prime \prime}+9 r^{-3} l^{\prime}+16 r^{-4} l\right) \phi .
\end{aligned}
$$

Thus plugging (3.10)-(3.12) into (3.9) gives the equation

$$
\begin{aligned}
0= & \left(l^{(4)}+14 r^{-1} l^{(3)}+r^{-2} l^{\prime \prime}\left(-2 \underline{\widetilde{\nabla}}^{*} \underline{\nabla}+51\right)\right. \\
& \left.+r^{-3} l^{\prime}\left(-10 \underline{\widetilde{\nabla}}^{*} \underline{\widetilde{\nabla}}+45\right)+r^{-4} l\left(\left(\underline{\widetilde{\nabla}}^{*} \widetilde{\nabla}\right)^{2}-8 \underline{\widetilde{\nabla}}^{*} \underline{\nabla}\right)\right) \phi .
\end{aligned}
$$

We now do the same for the cross component of (3.5). First of all from Propositions $2.20,2.26$ and 2.32 we see that this is the equation

$$
\begin{aligned}
0= & 4\left[-r^{-2} f^{\prime \prime}-r^{-3} f^{\prime}+r^{-4} f\left(\underline{\widetilde{\nabla}}^{*} \widetilde{\widetilde{\nabla}}+2\right)\right] \underline{\tilde{\delta}}(B)+8 r^{-4} f \underline{\widetilde{d} \widetilde{\mathrm{tr}}}(B) \\
+ & \left(k^{(4)}+6 r^{-1} k^{(3)}-r^{-2} k^{\prime \prime}\left(2 \underline{\widetilde{\nabla}}^{*} \underline{\widetilde{\nabla}}+9\right)-r^{-3} k^{\prime}\left(2 \underline{\widetilde{\nabla}}^{*} \underline{\widetilde{\nabla}}+15\right)\right. \\
& \left.+r^{-4} k\left(\left(\underline{\widetilde{\nabla}}^{*} \underline{\widetilde{\nabla}}\right)^{2}+16 \underline{\underline{\nabla}}{ }^{*} \underline{\widetilde{\nabla}}+28\right)\right) \tau-12 r^{-4} k \underline{\widetilde{d} \delta} \tau \\
& +4\left(r^{-2} l^{\prime \prime}+r^{-3} l^{\prime}-r^{-4} l\left(\underline{\widetilde{\nabla}}^{*} \underline{\nabla}+8\right)\right) \underline{\widetilde{d}} \phi .
\end{aligned}
$$


Now, using (3.7) we see that

$$
\begin{array}{r}
4\left[-r^{-2} f^{\prime \prime}-r^{-3} f^{\prime}+r^{-4} f\left(\underline{\widetilde{\nabla}}^{*} \underline{\nabla}+2\right)\right] \underline{\widetilde{\delta}}(B) \\
=4\left(r^{-1} k^{(3)}+7 r^{-2} k^{\prime \prime}+r^{-3} k^{\prime}\left(-\underline{\widetilde{\nabla}}^{*} \underline{\widetilde{\nabla}}+3\right)\right. \\
\left.-4 r^{-4} k\left(\underline{\widetilde{\nabla}}^{*} \underline{\widetilde{\nabla}}+2\right)\right) \tau .
\end{array}
$$

Next using (3.6) we make the simplification

$$
8 r^{-4} f \underline{\tilde{d} \widetilde{\mathrm{tr}}}(B)=-8 r^{-4} \underline{\tilde{d}} \phi,
$$

and using (3.8) we simplify

$$
-12 r^{-4} k \underline{\widetilde{d} \widetilde{\delta}} \tau=12\left(r^{-3} l^{\prime}+4 r^{-4} l\right) \underline{\tilde{d}} \phi .
$$

Plugging (3.15)-(3.17) into (3.14) gives

$$
\begin{aligned}
0= & \left(k^{(4)}+10 r^{-1} k^{(3)}+r^{-2} k^{\prime \prime}\left(-2 \underline{\widetilde{\nabla}}^{*} \underline{\widetilde{\nabla}}+19\right)\right. \\
& \left.-k^{\prime}\left(6 \underline{\widetilde{\nabla}}^{*} \underline{\nabla}+3\right)+r^{-4} k\left(\left(\underline{\widetilde{\nabla}}^{*} \underline{\widetilde{\nabla}}\right)^{2}-4\right)\right) \tau \\
& +4\left(r^{-2} l^{\prime \prime}+4 r^{-3} l^{\prime}+r^{-4} l\left(-\underline{\widetilde{\nabla}}^{*} \underline{\nabla}+2\right)\right) \underline{\widetilde{d}} \phi .
\end{aligned}
$$

Finally we need to simplify the horizontal component of (3.5). Again using Propositions $2.20,2.26$ and 2.32 we get the equation

$$
\begin{aligned}
& 0=\left(f^{(4)}+6 r^{-1} f^{(3)}-r^{-2} f^{\prime \prime}\left(1+2 \underline{\widetilde{\nabla}}^{*} \underline{\nabla}\right)\right. \\
& \left.-r^{-3} f^{\prime}\left(2 \underline{\widetilde{\nabla}}^{*} \underline{\nabla}+7\right)+r^{-4} f\left(\underline{\widetilde{\nabla}}^{*} \underline{\nabla}+2\right)^{2}\right) B \\
& -4 r^{-4} f \widetilde{\nabla}^{\operatorname{sym}} \widetilde{\tilde{\delta}}(B)+4 r^{-4} f \underline{\widetilde{\operatorname{tr}}}(B) \underline{\widetilde{g}} \\
& +4\left(r^{-2} k^{\prime \prime}+r^{-3} k^{\prime}-r^{-4} k\left(\underline{\widetilde{\nabla}}^{*} \underline{\widetilde{\nabla}}+5\right)\right)\left(\underline{\widetilde{\nabla}}^{\mathrm{sym}} \tau\right)-8 r^{-4} k \underline{\widetilde{\delta}} \tau \underline{\widetilde{g}} \\
& +4\left(r^{-2} l^{\prime \prime}+r^{-3} l^{\prime}-r^{-4} l\left(\underline{\widetilde{\nabla}}^{*} \underline{\widetilde{\nabla}}+4\right)\right)(\phi \underline{\widetilde{g}}) \\
& +4 r^{-4} l \underline{\widetilde{\nabla}}^{\mathrm{sym} \tilde{d}} \phi \text {. }
\end{aligned}
$$

First using (3.7) we simplify

$$
-4 r^{-4} f \underline{\widetilde{\nabla}}^{\mathrm{sym}} \widetilde{\widetilde{\delta}}(B)=4\left(r^{-3} k^{\prime}+4 r^{-4} k\right) \underline{\widetilde{\nabla}}^{\mathrm{sym}} \tau .
$$

Using (3.6) we simplify

$$
4 r^{-4} f \underline{\widetilde{\operatorname{tr}}}(B) \underline{\widetilde{g}}=-4 r^{-4} l \phi \underline{\widetilde{g}} .
$$

Next from (3.8) we simplify

$$
-8 r^{-4} k \underline{\widetilde{\delta}} \tau \underline{\widetilde{g}}=8\left(r^{-3} l^{\prime}+4 r^{-4} l\right) \phi \underline{\underline{g}} .
$$


Plugging (3.20)-(3.22) into (3.19) gives

$$
\begin{aligned}
0= & \left(f^{(4)}+6 r^{-1} f^{(3)}-r^{-2} f^{\prime \prime}\left(1+2 \underline{\widetilde{\nabla}}^{*} \widetilde{\nabla}\right)\right. \\
& \left.-r^{-3} f^{\prime}\left(2 \underline{\widetilde{\nabla}}^{*} \underline{\widetilde{\nabla}}+7\right)+r^{-4} f\left(\underline{\widetilde{\nabla}}^{*} \underline{\widetilde{\nabla}}+2\right)^{2}\right) B \\
& +4\left(r^{-2} k^{\prime \prime}+2 r^{-3} k^{\prime}-r^{-4} k\left(\underline{\widetilde{\nabla}}^{*} \widetilde{\nabla}+1\right)\right)\left(\underline{\widetilde{\nabla}}^{\text {sym }} \tau\right) \\
& +4\left(r^{-2} l^{\prime \prime}+3 r^{-3} l^{\prime}+r^{-4} l\left(-\underline{\widetilde{\nabla}}^{*} \underline{\widetilde{\nabla}}+3\right)\right) \phi \underline{\widetilde{g}}+4 r^{-4} l \underline{\widetilde{\nabla}}^{\mathrm{sym}} \underline{\underline{d}} \phi .
\end{aligned}
$$

Lemma 3.2. The system of equations (3.5) is equivalent to

$$
\begin{aligned}
& 0=\left(\left(f^{(4)}+6 r^{-1} f^{(3)}-r^{-2} f^{\prime \prime}\left(1+2 \underline{\nabla}^{*} \underline{\nabla}\right)\right.\right. \\
& \left.-r^{-3} f^{\prime}\left(2 \underline{\nabla}^{*} \underline{\widetilde{\nabla}}+7\right)+r^{-4} f\left(\underline{\widetilde{\nabla}}^{*} \underline{\widetilde{\nabla}}+2\right)^{2}\right) B \\
& +4\left(r^{-2} k^{\prime \prime}+2 r^{-3} k^{\prime}-r^{-4} k\left(\underline{\widetilde{\nabla}}^{*} \underline{\widetilde{\nabla}}+1\right)\right)\left(\underline{\widetilde{\nabla}}^{\text {sym }} \tau\right) \\
& +4\left(r^{-2} l^{\prime \prime}+3 r^{-3} l^{\prime}+r^{-4} l\left(-\underline{\widetilde{\nabla}}^{*} \underline{\widetilde{\nabla}}+3\right)\right) \phi \underline{\widetilde{g}}+4 r^{-4} l \underline{\widetilde{\nabla}}^{\mathrm{sym}} \underline{\tilde{d}} \phi, \\
& 0=\left(k^{(4)}+10 r^{-1} k^{(3)}+r^{-2} k^{\prime \prime}\left(-2 \underline{\widetilde{\nabla}}^{*} \underline{\widetilde{\nabla}}+19\right)\right. \\
& \left.-r^{-3} k^{\prime}\left(6 \underline{\widetilde{\nabla}}^{*} \underline{\tilde{\nabla}}+3\right)+r^{-4} k\left(\left(\underline{\widetilde{\nabla}}^{*} \underline{\widetilde{\nabla}}\right)^{2}-4\right)\right) \tau \\
& +4\left(r^{-2} l^{\prime \prime}+4 r^{-3} l^{\prime}+r^{-4} l\left(-\underline{\widetilde{\nabla}}^{*} \underline{\widetilde{\nabla}}+2\right)\right) \underline{\tilde{d}} \phi, \\
& 0=\left(l^{(4)}+14 r^{-1} l^{(3)}+r^{-2} l^{\prime \prime}\left(-2 \underline{\widetilde{\nabla}}^{*} \underline{\widetilde{\nabla}}+51\right)\right. \\
& \left.+r^{-3} l^{\prime}\left(-10 \underline{\widetilde{\nabla}}^{*} \underline{\widetilde{\nabla}}+45\right)+r^{-4} l\left(\left(\underline{\widetilde{\nabla}}^{*} \underline{\nabla}\right)^{2}-8 \underline{\widetilde{\nabla}}^{*} \underline{\nabla}\right)\right) \phi, \\
& 0=r^{-1} f \underline{\widetilde{\delta}}(B)+\left(k^{\prime}+4 r^{-1} k\right) \tau, \\
& 0=\left(l^{\prime}+4 r^{-1} l\right) \phi+r^{-1} k(\underline{\widetilde{\delta}} \tau) \text {. }
\end{aligned}
$$

We now analyse all solutions to this system of equations. We notice that for any solution, $l$ satisfies the determined ODE in (3.26). Thus we can compute the solutions to (3.26) in terms of the eigenvalues of the Laplacian on $S^{3}$ acting on functions. Once these are determined we notice that the equations (3.28) and (3.27) show that both $f$ and $k$ will have the same decay rate as $l$. Once this is done we restrict to the case where $l=0$. Here we notice that $k$ satisfies the now determined ODE in (3.25). We can classify these solutions in terms of the eigenvalues of the Laplacian on $S^{3}$ acting on one-forms. Once again we can use equation (3.28) to conclude that $f$ has the same decay rate as $k$. Finally we restrict to the case where $l=k=0$. Then it is clear that $f$ satisfies the determined ODE in (3.24), and we classify solutions in terms of the eigenvalues of the Laplacian on $S^{3}$ acting on traceless symmetric two-tensors. We now make this rigorous in a series of lemmas. 
Lemma 3.3. The solutions to 3.5 satisfying $l=k=0$ are

$$
h=\sum_{i} r^{b_{i}} B_{i}
$$

where either $b_{i}>0$ or

$$
b_{i} \leq-2 .
$$

Proof. Since $k=l=0$ we have $\underline{\tilde{\operatorname{tr}}} B=\underline{\widetilde{\delta}} B=0$. Say $\lambda$ is an eigenvalue for the Laplacian of $S^{3}$ acting on traceless symmetric two-tensors, and suppose $B_{\lambda}$ is in the eigenspace of $\lambda$. Equation (3.24) then reduces to an ODE with solutions

$$
r^{ \pm 1 \pm \sqrt{\lambda+3}} B_{\lambda} \text {. }
$$

From Lemma 6.1 we know that the smallest eigenvalue is 6 , so the result follows.

Lemma 3.4. The solutions to 3.5 satisfying $l=0$ are

$$
h=\sum_{i} r^{b_{i}}\left(B_{i}+\tau_{i} \otimes d r\right),
$$

where either $b_{i}>0$ or

$$
b_{i} \leq-2 .
$$

Proof. Note that since $l=0$, equation (3.27) implies that $\underline{\tilde{\delta}} \tau=0$. The eigenvalues for the Laplacian acting on 1-forms on $S^{3}$ are given by [7]

$$
a_{j}:=(j+1)(j+3) .
$$

Say $\tau_{j}$ is in the eigenspace of $a_{j}$. Then (3.25) reduces to an ODE with solutions

$$
r^{-2 \pm \sqrt{a_{j}+2}} \tau_{j}
$$

We point out that the decay rates are all less than -2 . Also, since the decay rate -4 does not occur, the expression $k^{\prime}+4 r^{-1} k$ never vanishes. Thus by equation (3.28) we see that $f=c r^{-2 \pm \sqrt{a_{j}+2}}$, and the result follows.

Lemma 3.5. The solutions to 3.5 satisfying $l \neq 0$ are

$$
h=L_{X} g_{0}+\sum_{i} r^{b_{i}}\left(B_{i}+\tau_{i} \otimes d r+\phi d r \otimes d r\right),
$$

where either $b_{i}>0$ or

$$
b_{i} \leq-2 .
$$

Proof. The eigenvalues of the Laplacian acting on functions on $S^{3}$ are

$$
a_{j}:=j(j+2) .
$$

Suppose $\phi_{j}$ is in the eigenspace of $a_{j}$. Equation (3.26) reduces to an ODE with solutions

$$
l(r)=r^{-2 \pm 1 \pm \sqrt{a_{j}+1}} .
$$

In the case where $l(r)=r^{-3 \pm \sqrt{a_{j}+1}}$, the solution to the whole system is given by a Lie derivative term, specifically $L_{X} g_{0}$, where

$$
X_{*}=r^{-1 \pm \sqrt{a_{j}+1}} d \phi_{j}+\left(-1 \pm \sqrt{a_{j}+1}\right) r^{-2 \pm \sqrt{a_{j}+1}} \phi_{j} d r .
$$


For the solutions $l(r)=r^{-1 \pm \sqrt{a_{j}+1}}$ we note that for $j>0$ the decay rates are always less than or equal to -2 . In the case $j=0$, i.e. where $\phi=c$, consider the radially parallel solution, i.e. where $l(r)=c$. Using equation (3.27) we see that $k$ is also a nonzero constant. Using this equation, (3.26) reduces to $\left(\left(\underline{\widetilde{\nabla}}^{*} \underline{\underline{\nabla}}\right)^{2}-4\right) \tau=$ 0 , so we see that this solution does not in fact occur since 2 is not an eigenvalue of the Laplacian on $S^{3}$ acting on one-forms.

In any of the cases above, using equation (3.6) we see that $f$ will have the same decay rate as $l$. Given this, equation (3.28) implies that $k$ must also have the same decay rate.

Together the above lemmas and earlier calculations prove the following proposition.

Proposition 3.6. On a flat cone, solutions of (3.1) satisfying $\delta h=0$ and $\Delta \operatorname{tr} h=$ 0 can be written uniquely as a sum

$$
h=L_{X} g_{0}+\sum_{i} r^{b_{i}}\left(B_{i}+\tau_{i} \otimes d r+\phi d r \otimes d r\right),
$$

where either $b_{i}>0$ or

$$
b_{i} \leq-2 .
$$

We hasten to point out that we will not in fact be able to guarantee the divergence-free condition. This is due to the presence of certain eigenvalues of the Laplace-Beltrami operator acting on one-forms. Thus we follow the technique used in [1] and consider a modified divergence-free condition. In particular, fix $t \neq 0$ with $|t|$ very small. Let

$$
\delta_{t}=\delta-t i_{r^{-1} \frac{\partial}{\partial r}} .
$$

We will be able to prescribe that $\delta_{t} h=0$ for $t$ arbitrarily small. Given this, we define the following modified equation:

$$
\begin{aligned}
0= & \Delta^{2} h-2 t \Delta \delta^{*} i_{r^{-1} \frac{\partial}{\partial r}} h \\
& +\frac{1}{3}\left(\nabla^{2} \Delta \operatorname{tr}_{g} h-\left(\Delta^{2} \operatorname{tr}_{g} h\right) g+t\left(\Delta \delta i_{r^{-1}} \frac{\partial}{\partial r} h\right) g+2 t \nabla^{2} \delta i_{r^{-1}} \frac{\partial}{\partial r} h\right) \\
:= & \mathcal{P}(h) .
\end{aligned}
$$

Following the analysis of Proposition 3.6 we can write the solutions satisfying $\Delta \operatorname{tr} h=0$ as growth and decay solutions where the rates are perturbations of those calculated above. In particular we can write

$$
h=r^{\beta_{i}} T_{i},
$$

where $T_{i}$ is some symmetric bilinear form and $\left\{T_{i}\right\}$ are orthonormal with respect to the inner product

$$
\left\langle\left\langle h_{1}, h_{2}\right\rangle\right\rangle=r^{-3} \int_{\left(r, S^{3}\right)}\left\langle h_{1}, h_{2}\right\rangle d V_{S^{3}} .
$$

We also have the following corollary.

Corollary 3.7. For $t$ sufficiently small, there are no radially parallel solutions of (3.37) satisfying $\Delta \operatorname{tr} h=0$. 
Proof. We note that the radially parallel solutions found above all had a $d r$ component, and so in the perturbed equation are no longer radially parallel. Thus the only possibility would be $f(r) B$, where $f(r)$ is a constant function and $B$ is trace-free and divergence-free. However, no such solution occurs according to our analysis above. Thus the corollary follows.

So, according to the above results, we can decompose any solution satisfying $\Delta \operatorname{tr} h=0$ as

$$
h=h_{\uparrow}+h_{\downarrow},
$$

where $h_{\uparrow}$ are the solutions with positive growth rate and likewise $h_{\downarrow}$ are solutions which decay in $r$. Now let

$$
\beta=\min _{i}\left|\beta_{i}\right|>0 .
$$

We can now state the first of our decay estimates. We start with some notation. For a fixed annulus $A_{a, b}(p)$ we have the norm

$$
\||| h||\|_{a, b}=\int_{a}^{b}\|h\|^{2} r^{-1} d r
$$

where the norm \|\| is defined in (3.39). This norm is defined so that if $w=$ $a^{-2} \psi_{a}^{*}(h)$, where $\psi_{a}$ is the natural scaling map $\psi_{a}(x, r)=(x, a r)$, then

$$
\left\|h\left|\left\|_{a, L a}=\right\|\right||w|\right\|_{1, L} .
$$

Corollary 3.8. Given $0<\beta^{\prime}<\beta$ there exists $l$ such that for all $a>0$ and $L \geq l$,

$$
\begin{gathered}
\left\|\left|h_{\uparrow}\right|\right\|_{L a, L^{2} a} \geq L^{\beta^{\prime}}\left\|h_{\uparrow}\right\|_{a, L a}, \\
\left\|\left|h_{\downarrow}\|\|_{L a, L^{2} a} \leq L^{-\beta^{\prime}}\left\|\mid h_{\downarrow}\right\| \|_{a, L a} .\right.\right.
\end{gathered}
$$

Lemma 3.9. Given $0<\beta^{\prime}<\beta$ there exists $L$ such that if $k$ is a solution to (3.37) satisfying $\delta_{t} k=0$ and $\Delta \operatorname{tr} k=0$, then if

$$
\left\|| | k \left|\left\|_{L, L^{2}} \geq L^{\beta^{\prime}}|||k|\right\|_{1, L}\right.\right.
$$

then

$$
\left\||| k\left|\|_{L^{2}, L^{3}} \geq L^{\beta^{\prime}}\right||| k||_{L, L^{2}},\right.
$$

and if

then

$$
\||k|\|_{L^{2}, L^{3}} \leq L^{-\beta^{\prime}}|||k| \|_{L, L^{2}}
$$

$$
\left\|| | k \left|\left\|_{L, L^{2}} \leq L^{-\beta^{\prime}}|||k|\right\|_{1, L} .\right.\right.
$$

\section{Asymptotic Curvature decay}

In this section we give the proof of Theorem 1.1. The proof will follow the techniques used in 1]. We first recall the definition of certain norms introduced in [1. Let $A_{u}$ denote the natural action of the scaling $\psi_{u}$ on tensors of type $(p, q)$, i.e.

$$
A_{u}=\left(\psi_{u^{-1}}\right)_{*} \otimes \cdots \otimes\left(\psi_{u^{-1}}\right)_{*} \otimes \psi_{u}^{*} \otimes \cdots \otimes \psi_{u}^{*} .
$$

Given $T$ a tensor of type $(p, q)$ we have

$$
|T(u, x)|_{k, \alpha ; 0}=\left|u^{p-q} A_{u} T(1, x)\right|_{k, \alpha ; 0},
$$


where the norm on the right is the $C^{k, \alpha}$-norm with respect to $g_{0}$ at the point $(1, x)$. Using this we define

$$
|T|_{k, \alpha ; 0}=\sup _{(u, x)}|T(u, x)|_{k, \alpha ; 0},
$$

and more generally

$$
|T|_{k, \alpha ; l}=\left|r^{-l} T\right|_{k, \alpha ; 0} .
$$

Given $T$ a tensor of type $(p, q)$ we write $T \in \mathcal{T}_{k, \alpha ; l}^{p, q}$ if $|T|_{k, \alpha ; l}<\infty$.

We will make use of a gauge-fixing theorem ([1], Theorem 3.1). For a given cone with cone-point $p$, let $A_{c, d}=\{(r, x) \mid c<r<d\}$.

Proposition 4.1. Fix $t \neq 0$. There exists $\kappa(t, k)$ such that if $\left(C\left(N^{n-1}\right), g_{0}\right)$ is a Ricci-flat cone and $g$ is a metric on $A_{c, d}(p) \subset C\left(N^{n-1}\right)$, where $d / c \geq 2$ such that

$$
\left|g-g_{0}\right|_{k, \alpha ; 0}<\kappa(t, k),
$$

then there exists a diffeomorphism $\phi: A_{c, d}(p) \rightarrow A_{c, d}(p)$ such that

$$
\phi^{*} g \in \mathcal{T}_{k, \alpha ; 0}^{0,2}
$$

and

$$
\delta_{t}\left(\phi^{*} g-g_{0}\right)=0 .
$$

Since we have assumed our given Bach-flat metric is asymptotically flat, we can use this theorem to construct gauges relative to the flat metric on the cone in the asymptotic regime. Specifically, let $g_{0}$ be the flat metric on $\mathbb{R}^{4}$ and let $g$ be a given Bach-flat metric on this cone. Consider a fixed annulus $A_{c, d}$ and let

$$
h=\phi^{*} g-g_{0},
$$

where $\phi$ is from Proposition 4.1 with respect to this fixed annulus. Then we note that $h$ satisfies

$$
\delta_{t} h=0
$$

and further

$$
B_{g_{0}+h}-B_{g_{0}}=0,
$$

which is a nonlinear elliptic equation on $h$ which we can think of as a perturbation of the linearized deformation equation for $h$ small. The following lemma makes this precise.

\section{Lemma 4.2.}

$$
B_{g_{0}+h}-B_{g_{0}}=\mathcal{P}(h)+F\left(h, g_{0}\right),
$$

where

$$
|F|_{k, \alpha ; 0} \leq C \sum_{p+q+r+s=4}\left|\nabla^{p} h\right|_{k-p, \alpha ;-p}\left|\nabla^{q} h\right|_{k-q, \alpha ;-q}\left|\nabla^{r} h\right|_{k-r, \alpha ;-r}\left|\nabla^{s} h\right|_{k-s, \alpha ;-s} .
$$

Also, since the metric $g$ is scalar-flat, we get the equation

$$
R_{g_{0}+h}-R_{g_{0}}=0
$$

from which we derive a similar lemma. 


\section{Lemma 4.3.}

$$
R_{g_{0}+h}-R_{g_{0}}=-\Delta \operatorname{tr} h-t \delta\left(i_{r^{-1} \frac{\partial}{\partial r}} h\right)+F^{\prime}\left(h, g_{0}\right)
$$

where

$$
\left|F^{\prime}\right|_{k, \alpha ; 0} \leq C \sum_{p+q=2}\left|\nabla^{p} h\right|_{k-p, \alpha ;-p}\left|\nabla^{q} h\right|_{k-q, \alpha ;-q} .
$$

Lemma 4.4. There exists a small constant $\chi(\Lambda, K)$ such that if $|h|_{k, \alpha ; 0} \leq \chi$, then

$$
|h(2 a)|_{k, \alpha ; 0} \leq\left. c(\Lambda, K, k)|||h|\right|_{a, 4 a} .
$$

Proof. Using the scale-invariance of the respective norms, it suffices to consider the case $a=1$. The result follows in this case by standard elliptic theory.

Now let $\pi$ denote orthogonal projection onto $\operatorname{ker}(\mathcal{P}(h))$ with respect to the inner product defining the norm ||||$\|_{L a, L^{2} a}$. Similar to (3.40) we can write

$$
\pi h=(\pi h)_{\uparrow}+(\pi h)_{\downarrow} .
$$

Proposition 4.5. There exists $\chi=\chi(\Lambda, K)>0$ and L large so that if $|h|_{k, \alpha ; 0}<\chi$, then if

$$
|||h|\left\|_{L a, L^{2} a} \geq L^{\beta^{\prime}}|||h|\right\|_{a, L a}
$$

then

$$
\||| h||_{L^{2} a, L^{3} a} \geq\left. L^{\beta^{\prime}}|||h|\right|_{L a, L^{2} a}
$$

then

$$
\left.\left\||| h||_{L^{2} a, L^{3} a} \leq L^{-\beta^{\prime}}\right\||h|\right|_{L a, L^{2} a},
$$

Moreover, at least one of (4.17) or (4.19) must hold.

Proof. Suppose one had a sequence of gauges $\phi_{i}$ and solutions $h_{i}$, where $\left|h_{i}\right|_{k, \alpha ; 0} \rightarrow$ 0 but none of the assertions of the proposition hold. By rescaling our solutions and using a compactness argument (see [1], Lemma 5.22) we can produce a solution to (3.37) satisfying $\Delta \operatorname{tr} h=0$, which contradicts Lemma 3.9.

Proof of Theorem 1.1. We will adopt the notation used in this section. In particular choose $\chi$ and $L$ as in Proposition 4.5. Let $g_{0}$ denote the standard flat metric on $\mathbb{R}^{4}$. Since we already know that the metric $g$ is ALE of order 0 , there exists $\Psi: \mathbb{R}^{4} \rightarrow X$ so that

$$
\left|\Psi^{*} g-g_{0}\right|_{k, \alpha ; 0}=o(r) .
$$

Now consider the sequence of annuli $A_{L^{i} a, L^{i+1} a}(0)$. Using (4.21) we may apply Proposition 4.1 and choose a $\delta_{t}$-free gauge for $\Psi^{*} g$ with respect to $g_{0}, \phi_{i}$, on each annulus. By pushing forward this is equivalent to finding a sequence of flat metrics $g_{i}$, where $\Psi^{*} g-g_{i}$ is $\delta_{t}$-free with respect to $g_{i}$,

$$
h_{i}:=\Psi^{*} g-g_{i} .
$$

We note that

$$
\lim _{i \rightarrow \infty}||\left|h_{i}\right| \|_{L^{i} a, L^{i+1} a}=0 .
$$


Indeed, if this were not the case, then inductively applying Proposition 4.5, and in particular using that (4.17) implies (4.5) we can contradict (4.21). Since for any of the flat metrics $g_{i}$, there are no radially parallel solutions to the linearized deformation equation, we can conclude that (4.20) holds for all $i$.

Also, by passing to a subsequence, we can assume that for some flat metric $g_{\infty}$ we have

$$
\lim _{j \rightarrow \infty}\left|g_{i}-g_{\infty}\right|_{k, \alpha^{\prime} ; 0}=0 \quad\left(\alpha^{\prime}<\alpha\right) .
$$

Using this together with (4.20) and Lemma 4.4 we conclude that

$$
\left|\Psi^{*} g-g_{\infty}\right|_{k, \alpha ; 0} \leq c r^{-\beta^{\prime}}
$$

This proves that $g$ is ALE of order $\beta^{\prime}$.

To prove the full claim we will use an inductive procedure choosing better and better gauges. First of all we point out that the decay estimate (4.23) in fact suffices to find a completely divergence-free global gauge $\phi$ (Remark 3.23 in [1]). So, let $h=\phi^{*} g-g_{0}$ be divergence-free. First we get a better estimate on the trace of $h$. In particular, by Lemma 4.3 we have

$$
\Delta \operatorname{tr} h=F^{\prime}(h)
$$

where the estimate (4.23) implies

$$
F^{\prime}(h) \in \mathcal{T}_{k-2, \alpha ;-2 \beta-2}^{0,2} .
$$

Applying the Greens function we can conclude the existence of a function $f$ so that

$$
\Delta f=F^{\prime}(h),
$$

where

$$
f \in \mathcal{T}_{k, \alpha ;-2 \beta}^{0,2} .
$$

In particular this implies the equation

$$
\Delta(\operatorname{tr} h-f)=0 .
$$

As a harmonic function on $\mathbb{R}^{4}$, we know that the decay rate is at least -2 ; thus

$$
\operatorname{tr} h-f \in \mathcal{T}_{k, \alpha ;-2}^{0,2} \text {. }
$$

This argument already can be inducted to show

$$
\operatorname{tr} h \in \mathcal{T}_{k, \alpha ; 2-\delta}^{0,2}
$$

for any $\delta>0$. We now show how to get the rest of the estimate. By Lemma 4.2 we have

$$
\mathcal{P}(h)=F(h),
$$

where $\mathcal{P}$ now refers to the case $t=0$. Using (4.23) we conclude

$$
F(h) \in \mathcal{T}_{k-4, \alpha ;-4 \beta-4}^{0,2} \text {. }
$$

Again using a Greens function, this decay is sufficient to conclude the existence of $h_{1}$ such that

$$
\mathcal{P} h_{1}=F(h),
$$

where

$$
h_{1} \in \mathcal{T}_{k, \alpha ;-4 \beta}^{0,2} .
$$


Thus $\mathcal{P}\left(h-h_{1}\right)=0$. Given our estimate on the trace of $h$ we can assume that $h-h_{1}$ is trace-free. We note that $\mathcal{P}$ acting on the traceless piece of $h-h_{1}$ also vanishes. Using Proposition 3.6 we can write

$$
h-h_{1}=L_{X} g_{0}+h_{2},
$$

where

$$
h_{2} \in \mathcal{T}_{k, \alpha ;-\min \{4 \beta, 2\}}^{0,2}
$$

and also

$$
X \in \mathcal{T}_{k+1, \alpha ; 1-\beta}^{1,0} .
$$

Let $K_{X}$ be the diffeomorphism generated by taking the flow of $X$ to time 1 . It is clear from the above estimates that

$$
K_{X}^{*}\left(g_{0}\right)-L_{X} g_{0} \in \mathcal{T}_{k, \alpha ;-\min \{4 \beta, 2\}}^{0,2} .
$$

Furthermore putting together the above estimates we get

$$
K_{-X}^{*} \phi^{*} g-g_{0} \in \mathcal{T}_{k, \alpha ;-\min \{4 \beta, 2\}}^{0,2} .
$$

If $4 \beta \geq 2$ we are done. If not, start over with $h=K_{-X}^{*} \phi^{*} g-g_{0}$ and proceed as above with $\beta$ replaced by $4 \beta-\epsilon$ for very small $\epsilon>0$. It is clear that by induction we get decay of any order $\tau<2$.

While decay of order $\tau<2$ suffices for the proof of Theorem 1.2, we can in fact adapt techniques used in [3], as well as the result of Theorem 1.2, to show decay of order $\tau=2 \sqrt{1}$ In particular, given a Bach-flat scalar-flat ALE space of order $2-\delta$, one can construct a $C^{1, \alpha}$ metric on the orbifold compactification $\hat{X}$ of $X$ given by $\hat{g}=r^{-4} g$, where $r$ denotes the distance function of the background Euclidean metric. This regularity is sufficient to weakly solve the negative constant Yamabe problem locally around the orbifold point on $\hat{X}$. Indeed, one can phrase the solution to this problem in terms of the minimizer of a convex functional, and so a minimizer exists by standard arguments. Call this new metric $\hat{g}^{\prime}$. By conformal invariance of the Bach-flat condition, $\hat{g}^{\prime}$ is Bach-flat, and moreover the hypotheses of Theorem 1.2 are satisfied for $\hat{g}^{\prime}$. Thus Theorem 1.2 implies that $\hat{g}^{\prime}$ extends as a smooth orbifold metric. This implies that $g$ itself has a smooth orbifold compactification. At this point the argument is the same as in [3], Proposition 13. We apply the Trudinger trick and rescale by the lowest eigenfunction of the Yamabe Laplacian of $\hat{g}^{\prime}$ to get a smooth orbifold whose scalar curvature does not change sign. In particular, by the strong maximum principle, the scalar curvature is positive everywhere. This implies that the orbifold Greens function of the conformally related metric exists, and moreover one has $g=G_{y}^{2} \hat{g}^{\prime}$. This Greens function takes the form

$$
G_{y}=\frac{1}{4 \pi^{2} \rho^{2}}+O(1)
$$

where $\rho=\frac{1}{r}$, so the result follows by using inverted normal coordinates around the point in $\hat{X} \backslash X$. We also note that in recent work Sophie Chen has shown the optimal decay rate for constant scalar curvature Kähler metrics and metrics with harmonic curvature [2].

\footnotetext{
${ }^{1}$ The author would like to thank Jeff Viaclovsky for pointing out this argument.
} 
Remark. The result holds assuming harmonic curvature instead of the Bach-flat condition. In particular, using the Bianchi identity equation (1.11) implies an equation of the form $\Delta \mathrm{Rc}=\mathrm{Rm} * \mathrm{Rm}$. The linearized deformation equation is the same as the one analyzed in section 3 , and so one can apply the argument in Theorem 1.1 to conclude that the metric is in fact ALE of order $\tau$ for any $\tau<2$. To get to $\tau=2$ one can apply the above argument except for one important caveat. The harmonic curvature equation is not conformally invariant. However, one can follow the proof of Theorem 1.2 using our known decay estimates for the curvature and conformal factor to prove the existence of a smooth orbifold compactification of the metric $g$. At this point the argument is the same as the above.

\section{Proof of Theorem 1.2}

Let $(X, d, x)$ and $B(x, 1) \backslash\{x\}$ be as in the statement of the theorem. We already know by Theorem 1.1 of $\left[9\right.$ that $B(x, 1)$ has a $C^{0}$-orbifold structure at $x$. Thus in particular, lifting to the universal cover we may suppose $x=0$ and $B(x, 1) \subset$ $D(0,1)$, where $D$ denotes the distance ball in the Euclidean metric. Let $g$ be this metric which is smooth away from the origin and $C^{0}$ on $B(0,1)$. Fix a constant $s<1$, and consider a sequence of annuli in the metric $g$,

$$
A_{i}:=A\left(s^{i+1}, s^{i}\right),
$$

and let $g_{i}^{\prime}=\frac{1}{s^{4 i}} g$ be an inverted metric on $A_{i}$. Let $\rho_{i}$ denote the distance function of the metric $g_{i}^{\prime}$ on the annulus $A_{i}^{\prime}$. Since we have assumed a bound on the Sobolev constant, the $L^{2}$-norm of curvature, and that the first Betti number is bounded, we may apply the analysis of section 4 in 9 . In particular we may conclude that $\rho_{i} \rightarrow r$ as $i \rightarrow \infty$. In particular the annuli $A_{i}^{\prime}$ are approaching the standard annuli in $\mathbb{R}^{4}$, and since the scalar curvature of $g$ is bounded we get convergence to a scalarflat, Bach-flat ALE end of order 0. Thus the asymptotic analysis of section 4 above applies, so we conclude that this metric is ALE of order $\tau$ for any $\tau<2$. In terms of the metric $g$ this estimate yields

$$
g=\delta_{i j}+\mathcal{O}\left(r^{2-\delta}\right)
$$

where $r$ denotes distance from the origin. For $\delta$ sufficiently small this implies that the metric has a $C^{1, \alpha}$ extension through the origin. Using results from [5] this implies the existence of a harmonic coordinate around the origin. A simple computation using the Bianchi identity shows that the Bach-flat condition implies that the curvature satisfies an equation of the form $\Delta \mathrm{Rc}=\mathrm{Rm} * \mathrm{Rc}$. Thus we view the Bach-flat equation in harmonic coordinates as the system

$$
\begin{gathered}
\Delta \mathrm{Rc}=\mathrm{Rm} * \mathrm{Rc}, \\
\Delta g=\mathrm{Rc}+Q(g, \partial g) .
\end{gathered}
$$

Using the curvature estimate $|\mathrm{Rm}|=\mathcal{O}\left(r^{-\delta}\right)$ it follows from equation (5.2) that $\mathrm{Rc} \in W^{2, p}$ for any $p$. Using equation (5.3) it is clear that $g \in W^{3, p}$. This allows us to bootstrap and conclude that $g \in C^{\infty}$.

Remark. Again we point out that the result holds assuming harmonic curvature instead of the Bach-flat condition. The crucial step in the above argument is the curvature decay rate, and we mentioned after the proof of Theorem 1.1 that metrics with harmonic curvature satisfy this estimate. 


\section{Appendix: Analysis on the threE-Sphere}

Lemma 6.1. Let $\left(S^{3}, g\right)$ be the round three-sphere. The smallest eigenvalue of the rough Laplacian acting on traceless divergence-free symmetric two-tensors is 6 .

Proof. Using the identification of $S^{3}$ with $S U(2)$ we consider the standard global left-invariant Milnor frame $X^{1}, X^{2}, X^{3}$ with structure constants

$$
C_{23}^{1}=C_{31}^{2}=C_{12}^{3}=-2 .
$$

If $e_{1}, e_{2}, e_{3}$ denotes the corresponding coframe, we have the equation

$$
\nabla_{X^{i}} e_{j}=-e_{k} \sigma(i j k)
$$

where $\sigma(i j k)$ denotes the sign of the permutation $(i j k)$ and is zero if any of $i, j$ and $k$ are equal. Using this global frame we may write any traceless symmetric two-tensor as

$$
B_{i j} e_{i} \otimes e_{j}
$$

and compute

$$
\begin{aligned}
\nabla^{*} \nabla B= & -B_{k l, i i} e_{k} \otimes e_{l}-2 B_{k l, i}\left(\nabla_{X^{i}} e_{k} \otimes e_{l}+e_{k} \nabla \nabla_{X^{i}} e_{l}\right) \\
& -B_{k l}\left(\nabla_{X_{i}} \nabla_{X_{i}} e_{k} \nabla e_{l}+2 \nabla_{X^{i}} e_{k} \nabla \nabla_{X^{i}} e_{l}+e_{k} \nabla \nabla_{X^{i}} \nabla_{X^{i}} e_{l}\right) .
\end{aligned}
$$

Now a basic calculation using (6.1) shows that

$$
\nabla_{X^{i}} \nabla_{X^{i}} e_{k}=-2 e_{k}
$$

and similarly, using that $B$ is traceless it is easy to compute

$$
2 B_{k l} \nabla_{X^{i}} e_{k} \otimes \nabla_{X^{i}} e_{l}=-2 B_{k l} e_{k} \otimes e_{l} .
$$

Using these calculations and the fact that the smallest eigenvalue must occur when the coefficient functions are constant, the result follows.

Lemma 6.2. Let $\left(S^{3}, g\right)$ be the round three-sphere. Then given a function $\phi \in$ $C^{\infty}\left(S^{3}\right)$

$$
d \nabla^{*} \nabla \phi=\nabla^{*} \nabla d \phi+2 d \phi
$$

and

$$
\delta\left(\nabla^{*} \nabla\right) d \phi=-\left(\nabla^{*} \nabla\right)^{2} \phi+2 \nabla^{*} \nabla \phi
$$

and given a one-form $\alpha \in \wedge^{1} T^{*} S^{3}$,

$$
\delta\left(\nabla^{*} \nabla\right) \alpha=\nabla^{*} \nabla \delta \alpha-2 \delta \alpha
$$

and

$$
\delta\left(\nabla^{*} \nabla\right)^{2} \alpha=\left(\nabla^{*} \nabla\right)^{2} \delta \alpha-4 \nabla^{*} \nabla \delta \alpha+4 \delta \alpha
$$

and

$$
\delta L_{\alpha^{*}} g=-\nabla^{*} \nabla \alpha+\nabla \delta \alpha+2 \alpha
$$

and

$$
L_{\nabla^{*} \nabla} g=\nabla^{*} \nabla L_{\tau} g+4 L_{\tau} g-2 \delta \tau g
$$

and given $B$ a traceless symmetric two-tensor,

$$
\delta \nabla^{*} \nabla B=\nabla^{*} \nabla \delta B-4 \delta B+2 d \operatorname{tr} B
$$


and

$$
\delta\left(\nabla^{*} \nabla\right)^{2}=\left(\nabla^{*} \nabla\right)^{2} \delta B-8 \nabla^{*} \nabla \delta B+16 \delta B .
$$

Proof. We use the convention that $R_{i j i j}>0$, and more specifically choosing normal coordinates at one point we have

$$
\begin{aligned}
R_{i j k l} & =\left(g_{i k} g_{j l}-g_{j k} g_{i l}\right), \\
R_{i k} & =2 g_{i k}, \\
R & =6 .
\end{aligned}
$$

First for a function $\phi$ we compute

$$
\begin{aligned}
d \nabla^{*} \nabla \phi & =-\nabla_{i} \nabla^{k} \nabla_{k} \phi \\
& =-\nabla^{k} \nabla_{i} \nabla_{k} \phi-R_{i k k l} \nabla^{l} \phi \\
& =-\nabla^{k} \nabla_{k} \nabla_{i} \phi+2 \nabla_{i} \phi \\
& =\nabla^{*} \nabla d \phi+2 d \phi .
\end{aligned}
$$

Next

$$
\begin{aligned}
\delta\left(\nabla^{*} \nabla\right) d \phi & =-\nabla^{i} \nabla^{j} \nabla_{j} \nabla_{i} \phi \\
& =-\nabla^{i} \nabla^{j} \nabla_{i} \nabla_{j} \phi \\
& =-\nabla^{i}\left(\nabla_{i} \nabla^{j} \nabla_{j} \phi+R_{j i j k} \nabla_{k} \phi\right) \\
& =-\left(\nabla^{*} \nabla\right)^{2} \phi-R_{j i j k} \nabla^{i} \nabla_{k} \phi \\
& =-\left(\nabla^{*} \nabla\right)^{2} \phi+2 \nabla^{*} \nabla \phi .
\end{aligned}
$$

In the fourth line we used that the curvature is parallel on $S^{3}$. Next we compute

$$
\begin{aligned}
\delta\left(\nabla^{*} \nabla\right) \alpha & =-\nabla^{i} \nabla^{j} \nabla_{j} \alpha_{i} \\
& =-\nabla^{j} \nabla_{i} \nabla_{j} \alpha_{i}-R_{i j j k} \nabla^{k} \alpha_{i}-R_{i j i k} \nabla_{j} \alpha^{k} \\
& =-\nabla^{j} \nabla_{i} \nabla_{j} \alpha_{i} \\
& =-\nabla^{j}\left(\nabla_{j} \nabla_{i} \alpha_{i}+R_{i j i k} \alpha^{k}\right) \\
& =\nabla^{*} \nabla \delta \alpha-R_{i j i k} \nabla^{j} \alpha^{k} \\
& =\nabla^{*} \nabla \delta \alpha-2 \delta \alpha,
\end{aligned}
$$

and again we have used that the curvature is parallel. Using this computation we have

$$
\begin{aligned}
\delta\left(\nabla^{*} \nabla\right)^{2} \alpha & =\nabla^{*} \nabla \delta \nabla^{*} \nabla \alpha-2 \delta \nabla^{*} \nabla \alpha \\
& =\nabla^{*} \nabla\left(\nabla^{*} \nabla \delta \alpha-2 \delta \alpha\right)-2\left(\nabla^{*} \nabla \delta \alpha-2 \delta \alpha\right) \\
& =\left(\nabla^{*} \nabla\right)^{2} \delta \alpha-4 \nabla^{*} \nabla \delta \alpha+4 \delta \alpha
\end{aligned}
$$

Also we compute

$$
\begin{aligned}
\delta L_{\alpha^{*}} g & =\nabla^{i}\left(\nabla_{i} \alpha_{j}+\nabla_{j} \alpha_{i}\right) \\
& =-\nabla^{*} \nabla \alpha+\nabla_{j} \nabla^{i} \alpha_{i}+R_{i j i l} \alpha^{l} \\
& =-\nabla^{*} \nabla \alpha+\nabla \delta \alpha+2 \alpha .
\end{aligned}
$$


Finally we compute

$$
\begin{aligned}
L_{\nabla^{*} \nabla \tau} g= & -\nabla_{i} \nabla^{k} \nabla_{k} \tau_{j}-\nabla_{j} \nabla^{k} \nabla_{k} \tau_{i} \\
= & -\nabla^{k} \nabla_{i} \nabla_{k} \tau_{j}-R_{i k k l} \nabla^{l} \tau_{j}-R_{i k j l} \nabla^{k} \tau^{l} \\
& -\nabla^{k} \nabla_{j} \nabla_{k} \tau_{i}-R_{j k k l} \nabla^{l} \tau_{i}-R_{j k i l} \nabla^{k} \tau^{l} \\
= & -\nabla^{k} \nabla^{k} \nabla_{i} \tau_{j}-2 R_{i k j l} \nabla^{k} \tau^{l}+2 \nabla_{i} \tau_{j} \\
& -\nabla^{k} \nabla^{k} \nabla_{j} \tau_{i}-2 R_{j k i l} \nabla^{k} \tau^{l}+2 \nabla_{j} \tau_{i} \\
= & \nabla^{*} \nabla L_{\tau} g+4 L_{\tau} g-2 \delta \tau g .
\end{aligned}
$$

Now for $B$ a symmetric two-tensor,

$$
\begin{aligned}
\delta \nabla^{*} \nabla B & =-\nabla^{i} \nabla^{j} \nabla_{j} B_{i k} \\
& =-\nabla^{j} \nabla^{i} \nabla_{j} B_{i k}-R_{i j j l} \nabla^{l} B_{i k}-R_{i j i l} \nabla^{j} B_{k}^{l}-R_{i j k l} \nabla^{j} B_{i}^{l} \\
& =-\nabla^{j} \nabla^{i} \nabla_{j} B_{i k}-R_{i j k l} \nabla^{j} B_{i}^{l} \\
& =-\nabla^{j} \nabla^{i} \nabla_{j} B_{i k}-\delta B+d \operatorname{tr} B \\
& =-\nabla^{j}\left(\nabla_{j} \nabla^{i} B_{i j}+R_{i j i l} B_{k}^{l}+R_{i j k l} B_{i}^{l}\right)-\delta B+d \operatorname{tr} B \\
& =\nabla^{*} \nabla \delta B-R_{i j i l} \nabla^{j} B_{k}^{l}-R_{i j k l} \nabla^{j} B_{i l}-\delta B+d \operatorname{tr} B \\
& =\nabla^{*} \nabla \delta B-4 \delta B+2 d \operatorname{tr} B .
\end{aligned}
$$

Using this computation we have

$$
\begin{aligned}
\delta\left(\nabla^{*} \nabla\right)^{2} & =\nabla^{*} \nabla \delta \nabla^{*} \nabla B-4 \delta \nabla^{*} \nabla B \\
& =\nabla^{*} \nabla\left(\nabla^{*} \nabla \delta B-4 \delta B\right)-4\left(\nabla^{*} \nabla \delta B-4 \delta B\right) \\
& =\left(\nabla^{*} \nabla\right)^{2} \delta B-8 \nabla^{*} \nabla \delta B+16 \delta B .
\end{aligned}
$$

\section{ACKNOWLEDGEMEnTS}

The author would like to express deep gratitude to Gang Tian for suggesting this problem and for suggesting that the arguments of [1] could be used to prove Theorem 1.2. He would also like to thank Jeff Viaclovsky for interesting discussions related to this work. The author was partially supported by the NSF by DMS0703660 .

\section{REFERENCES}

[1] J. Cheeger and G. Tian, On the cone structure at infinity of Ricci flat manifolds with Euclidean volume growth and quadratic curvature decay, Invent. Math. 118 (1994) 493-571. MR1296356 (95m:53051)

[2] S. Chen, Optimal curvature decays on asymptotically locally euclidean manifolds, preprint.

[3] X.X. Chen, C. Lebrun and B. Weber, On conformally Kähler, Einstein manifolds, J. Amer. Math. Soc. 21 (2008), no. 4, 1137-1168. MR2425183

[4] A. Derdziński, Self-dual Kähler manifolds and Einstein manifolds of dimension four, Compositio Math. 49 (1983), no. 3, 405-433. MR707181 (84h:53060)

[5] D. M. DeTurck and J. L. Kazdan, Some regularity theorems in Riemannian geometry, Ann. Sci. École Norm. Sup. (4) 14 (1981) no. 3, 249-260. MR83f:53018

[6] A. L. Besse, Einstein manifolds, Springer-Verlag, Berlin, 1987. MR.867684 (88f:53087)

[7] S. Gallot and D. Meyer, Opérateur de courbure et Laplacien des formes différentielles d'une variété Riemannienne, J. Math. Pures et Appl. 54 (1975), no. 3, 259-284. MR0454884 $(56: 13128)$ 
[8] G. Tian and J. Viaclovsky, Moduli spaces of critical Riemannian metrics in dimension four, arXiv:math.DG/0312318.

[9] G. Tian and J. Viaclovsky, Bach-flat asymptotically locally Euclidean metrics, arXiv:math.DG/0310302.

Department of Mathematics, Fine Hall, Princeton University, Princeton, New JerSEY 08544

E-mail address: jstreets@math.princeton.edu 